\title{
COMPARAÇÃO DA QUALIDADE DE VIDA DE INDIVÍDUOS COM E SEM HANSENÍASE
}

\section{COMPARISON OF THE QUALITY OF LIFE OF INDIVIDUALS WITH AND WITHOUT LEPROSY}

Denise Carvalho Torres ${ }^{1}$; Kelyanne dos Santos Pinho²; Claudio Karone da Silva Borges ${ }^{3}$, Anderson Batista Lopes ${ }^{4}$, Gabriel Pereira da Costa ${ }^{5}$; Maria Claudia Gonçalves ${ }^{6}$; Sarah Tarcísia Rebelo Ferreira de Carvalho ${ }^{7}$

RESUMO: A Hanseníase é uma doença infectocontagiosa crônica que provoca disfunções no sistema somatossensorial, deficiência física, distúrbios psicológicos e exclusão social, podendo comprometer a qualidade de vida. O presente estudo tem como objetivo comparar a qualidade de vida de indivíduos com o diagnóstico de hanseníase e sem histórico de hanseníase. Trata-se de um estudo analítico do tipo transversal, com 100 indivíduos maiores de 18 anos, de ambos os gêneros. Os indivíduos foram divididos em dois grupos: GH-com 50 pacientes com diagnóstico de hanseníase que estão em tratamento assistido e GC-com 50 indivíduos sem histórico de hanseníase. Foram coletadas informações sociodemógraficas e de qualidade de vida, através do questionário SF-36 (Medical Outcome Study-Mos Short From Health Survey). O estudo foi aprovado pelo Comitê de Ética em Pesquisa (Parecer Número: 1.674.308). Constatou-se que o GH e o GC eram homogêneos estatisticamente em relação à idade, sexo e IMC (p > 0,05), sendo a maioria adulta jovens, do sexo masculino e peso considerado normal. Quanto à qualidade de vida, constatou-se, que o GH apresentou todos os oitos domínios significativamente pior em relação ao $\mathrm{GC}(\mathrm{p}<0,005)$. No GH, os domínios com escores mais baixos foram: aspectos físicos (escore=20,00); dor (escore=38,66); aspectos emocionais (escore =41,23) e; estado geral da saúde (escore=43,64). Considera-se que estes dados podem direcionar os profissionais de saúde envolvidos no tratamento

\footnotetext{
${ }^{1}$ Discente do curso de Fisioterapia da Universidade Ceuma. E-mail: denise_torres@outlook.com ${ }^{2}$ Discente do curso de Fisioterapia da Universidade Ceuma. E-mail: kel.pinho7@gmail.com 3Discente do curso de Fisioterapia da Universidade Ceuma. E-mail: claudio_karone@ hotmail.com

${ }^{4}$ Discente do curso de Fisioterapia da Universidade Ceuma. E-mail: andersobatista20@gmail.com

${ }^{5}$ Discente do curso de Fisioterapia da Universidade Ceuma. E-mail: gabrielcosta17@ hotmail.com

${ }^{6}$ Professora Doutora do Curso de Fisioterapia e do Programa de Mestrado em Meio Ambiente da Universidade Ceuma. E-mail: mcgfisio0@gmail.com

${ }^{7}$ Professora Doutora do Curso de Fisioterapia da Universidade Ceuma. E-mail: sarahtrfc@ hotmail.com
} 
de pacientes com hanseníase, no sentido de conscientizá-los de que esta doença envolve fatores físicos, psicológicos, mentais e sociais, devendo-se tratar não apenas os seus sintomas, mas os indivíduos integralmente.

PALAVRAS-CHAVE: Hanseníase; Qualidade de vida; Saúde.

ABSTRACT: Leprosy is a chronic infectious contagious disease that causes dysfunction in the somatosensory system, physical disability, psychological disturbances and social exclusion, which can compromise the quality of life. The present study aims to compare the quality of life of individuals with the diagnosis of leprosy and without a history of leprosy. This is an analytical cross-sectional study with 100 individuals older than 18 years, of both genders. Subjects were divided into two groups: GH-with 50 patients diagnosed with leprosy who are on assisted treatment and CG-with 50 individuals with no history of leprosy. Sociodemographic and quality of life information were collected through the SF-36 (Medical Outcome Study-Mos Short From Health Survey). The study was approved by the Research Ethics Committee (Opinion No. 1,674,308). GH and CG were statistically homogeneous in relation to age, sex and BMI ( $p>0.05$ ), with the majority of young adults being males and considered as normal. Regarding quality of life, it was found that the GH presented all eight domains significantly worse than the CG ( $\mathrm{p}<0.005)$. In $\mathrm{GH}$, the domains with lower scores were: physical aspects (score $=20.00$ ); pain (score $=38.66$ ); emotional aspects ( score $=41.23$ ) and; general health status (score $=43.64)$. It is considered that these data can direct health professionals involved in the treatment of patients with leprosy, in order to make them aware that this disease involves physical, psychological, mental and social factors, not only treating their symptoms, but individuals integrally.

KEYWORDS: Leprosy; Quality of life; Health. 


\section{Introdução}

A hanseníase é uma doença infectocontagiosa crônica, curável, causada pelo bacilo Mycobacterium leprae. Possui alta contagiosidade e baixa morbidade, afetando principalmente os nervos periféricos e os nervos superficiais da pele, podendo atingir olhos e órgãos internos (BRASIL, 2015a; LASTÓRIA; ABREU, 2012; VÉRAS et al., 2012).

O Brasil ocupa o segundo lugar no número absoluto de casos de hanseníase, perdendo apenas para índia. O registro ativo de hanseníase no país indica maior concentração desses casos nas regiões norte, nordeste e centro-oeste. No Nordeste, a maior taxa de prevalência e incidência se encontra no estado do maranhão (BRASIL, 2015b; BRASIL, 2014; BRITO et al., 2016).

Historicamente, a hanseníase sempre esteve estigmatizada enquanto praga ou castigo divino, pautada em um contexto excludente, baseada em fábulas do imaginário popular. Assim, o acompanhamento terapêutico para o paciente hansênico esteve durante muitos anos negligenciado a cuidados paliativos, sendo o isolamento de contado a única medida profilática realizada (AVELLEIRA et al., 2014).

O diagnóstico da hanseníase é essencialmente clínico, feito por meio de exames dermatoneurológico e coleta da história do paciente. Faz-se também o exame laboratorial para verificação de presença de bacilos, sendo que a negatividade do exame não exclui o diagnóstico de hanseníase. Quanto mais rápido o diagnóstico, melhor o prognóstico do paciente (BRASIL, 2010; BRASIL, 2015a).

O Ministério da Saúde considera como caso de hanseníase para tratamento aqueles pacientes que possuem um ou mais dos seguintes achados: espessamento de tronco nervoso, alteração de sensibilidade ou baciloscopia positiva na pele (ARAÚJO et al., 2016).

Desde 1995, o tratamento da hanseníase é oferecido gratuitamente no sistema público de saúde brasileiro, tanto para medidas medicamentosas, quanto para reabilitação física e psicossocial (OPROMOLLA et al., 2011). No entanto, foi a partir de 2010, através da portaria $\mathrm{n}^{\circ} 3.125$, que trata das Diretrizes para Vigilância, atenção e controle da hanseníase, que o tratamento para esta doença foi normatizado pelo Ministério da Saúde (BRASIL, 2010).

O tratamento da hanseníase é ambulatorial, realizado nos serviços básicos de saúde, com administração da poliquimioterapia (PQT/OMS), capaz de matar o bacilo e, assim, evitar a evolução da doença, prevenindo as incapacidades e deformidades consequentes, e levando o paciente à cura (BRASIL, 2010). 
Após o término do tratamento realizado adequadamente, em geral com uso de poliquimioterapia por seis a doze meses, pode-se considerar o paciente como curado, sendo o mesmo retirado das estatísticas oficiais. Desta forma, mesmo que permaneçam com alguma sequela da doença ou apresentem episódios reacionais, esses pacientes não são mais considerados como casos de hanseníase (RAMOS; SOUTO, 2010).

Destaca-se que 23,3\% dos pacientes diagnosticados com hanseníase apresentam grau de incapacidade nível I e II. Concluindo assim, que quando diagnosticados, muitos dos pacientes já apresentam importantes sequelas incapacitantes (BARBOSA et al., 2016; SILVA, 2013).

Desta forma, mesmo após o início do tratamento, alguns indivíduos com hanseníase podem apresentar incapacidades decorrentes do diagnóstico tardio, com consequente interferência na qualidade de vida, diminuição da capacidade de trabalho, limitação da vida social, problemas psicológicos, entre outros fatores (UCHÔA et al., 2015; MONTEIRO et al., 2013).

A qualidade de vida é um termo amplo, influenciado por diversos fatores ligados ao bemestar que podem ser analisados sob muitos aspectos, incluindo: físico, material, social, emocional e produtivo. Neste sentido, a qualidade de vida indica a percepção do indivíduo acerca de sua vida social, cultural e emocional (NÓBREGA; SARAIVA, 2003).

As avaliações de qualidade de vida são essenciais para o adequado planejamento e acompanhamento do tratamento, para a identificação dos fatores preditores de qualidade de vida, como também para o desenvolvimento de estratégias de promoção de bem-estar e prevenção de saúde mental (HENRY et al., 2003). Para tanto, a literatura indica diversos questionários padronizados, dentre os quais se destaca o Short Form Health Survey - 36 (SF-36), instrumento validado que avalia a qualidade de vida através de oito domínios, a saber: capacidade funcional, limitação por aspectos físicos, dor, estado geral de saúde, vitalidade, aspectos sociais, aspectos emocionais e saúde mental (VITORINO, 2004).

Neste contexto, destacando a multidimensionalidade da hanseníase, percebe-se a importância de avaliar a qualidade de vida de pacientes com esta doença, para poder identificar a influência da mesma em diversos aspectos do indivíduo e desta forma, agregar novos conceitos para as ações do autocuidado integral para com o paciente.

Diante do exposto, o presente estudo tem o objetivo de comparar a qualidade de vida de indivíduos com diagnóstico de hanseníase que estão em tratamento assistido e indivíduos sem histórico de hanseníase. 


\section{Materiais e métodos}

Trata-se de um estudo observacional analítico do tipo transversal, realizado com 100 indivíduos maiores de 18 anos, de ambos os gêneros, que aceitaram participar do estudo voluntariamente mediante assinatura do Termo de Consentimento Livre e Esclarecido - TCLE (Apêndice 1).

Os participantes foram divididos em dois grupos: Grupo Hanseníase Tratamento (GH) com 50 indivíduos com diagnóstico de hanseníase em tratamento assistido e; Grupo Controle (GC) com 50 indivíduos que não apresentam histórico de hanseníase.

Para o GH, foram selecionados pacientes que apresentavam diagnóstico clínicolaboratorial de hanseníase definido pelo médico, de acordo com os critérios do Programa Nacional de Controle de Hanseníase, independente da forma clínica, gênero e tempo de diagnóstico, e que estivessem realizando tratamento assistido de hanseníase no Centro de Saúde de Fatima ou no Hospital Aquiles Lisboa, ambos no município de São Luís-MA.

Já no GC, foram inclusos 50 indivíduos, dentre discentes, usuários e funcionários da clínica escola da Universidade Ceuma e acompanhantes de pacientes do Hospital Aquiles Lisboa, que não apresentavam histórico de hanseníase.

Foram excluídos dos dois grupos citados, os participantes que apresentaram distúrbio mental e patologias cardíacas diagnosticadas; distúrbios neurológicos ou doenças crônicas não controladas, exceto neuropatia periférica sensitiva decorrente da hanseníase no $\mathrm{GH}$; e que estivessem fazendo uso de medicamentos psicotrópicos, além de grávidas; indivíduos com IMC $\geq 35$, indicando obesidade grau II ou superior; pacientes com comprometimento total da visão e com doenças vestibulares diagnosticadas.

Para a avaliação da qualidade de vida, foi aplicado o questionário SF36 - Medical Outcome Study-Mos Short From Health Survey (Anexo 1). Este instrumento consiste em um questionário genérico que foi traduzido e validado para a realidade Brasileira, contendo 11 perguntas, dividido em 8 domínios, a saber: capacidade funcional, limitação por aspectos físicos, dor, estado geral de saúde, vitalidade, aspectos sociais, aspectos emocionais e saúde mental. Todos esses domínios representam os valores relevantes da qualidade de vida. Assim, para cada domínio, o indivíduo é pontuado de zero, indicando a pior saúde, a cem, em relação ao melhor estado de saúde possível (CICONELLI, 2003).

A análise de dados foi realizada mediante o programa SPSS versão 19. Na estatística descritiva, as variáveis quantitativas foram apresentadas por média, mínima, máxima e desvio 
padrão, enquanto as variáveis qualitativas foram descritas através de frequências absolutas e relativas. Para avaliação de possíveis diferenças na frequência do gênero entre os grupos com hanseníase e controle foi empregado o teste do Qui-quadrado. Já para comparações entre as médias de idade e IMC entre os grupos avaliados foi utilizado o teste de ANOVA one-way, onde previamente se analisou os pressupostos de normalidade (Teste de Shapiro-Wilk) e homogeneidade de variância (Teste de Levene) dos dados quantitativos. A comparação entre as médias dos aspectos da qualidade de vida nos diferentes grupos e entre os gêneros (para o grupo de hanseníase) foi realizada através do teste de ANOVA one-way. Em todas as análises, foi adotado nível de significância de 0,05 .

Este estudo seguiu todas as recomendações da resolução 466/2012 do Conselho Nacional de Saúde que regulamenta as pesquisas envolvendo seres humano, tendo sido aprovada pelo Comitê de Ética - Parecer Número: 1.674.308. Destaca-se que a pesquisa foi financiada pela Fundação de Amparo à Pesquisa e ao Desenvolvimento do Maranhão - FAPEMA (Edital Universal nº 05/2015).

\section{Resultados}

Esta pesquisa foi realizada com o objetivo de comparar a qualidade de vida de indivíduos com e sem hanseníase, sendo realizada com 100 indivíduos, sendo 50 do Grupo Hanseníase (GH) e 50 do Grupo Controle (GC).

Em relação à idade, o GH apresentou uma média de 38,62ะ12,57 anos, sendo mínima de

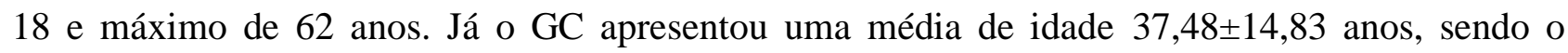
mínimo de 18 e máxima de 63 anos. No entanto, essa diferença não mostrou significância estatística $(\mathrm{p}=0,67937)$.

Ambos os grupos também eram homogêneos estatisticamente em relação às variáveis sexo, e IMC ( $p$ > 0,05). A maioria dos dois grupos era do sexo masculino, predominando em ambos os grupos indivíduos com peso considerado normal (Tabela 1).

Tabela 1 - Características sociodemográficas de indivíduos do grupo hanseníase e do grupo controle. São Luís - MA, 2017.

\begin{tabular}{|c|c|c|c|c|c|c|}
\hline \multirow{2}{*}{\multicolumn{2}{|c|}{ Características sóciodemográficas }} & \multicolumn{2}{|c|}{ Grupo Hanseníase } & \multicolumn{2}{|c|}{ Grupo Controle } & \multirow[b]{2}{*}{$p$} \\
\hline & & $\mathrm{N}$ & $\%$ & $\mathrm{~N}$ & $\%$ & \\
\hline \multirow{2}{*}{ Sexo } & Masculino & 36 & 72,0 & 34 & 68,0 & \multirow{2}{*}{0,663} \\
\hline & Feminino & 14 & 28,0 & 16 & 32,0 & \\
\hline \multirow{4}{*}{ IMC } & Baixo peso & 01 & 02,0 & 01 & 02,0 & \multirow{4}{*}{0,60683} \\
\hline & Peso normal & 25 & 50,0 & 22 & 44,0 & \\
\hline & Sobrepeso & 19 & 38,0 & 21 & 42,0 & \\
\hline & Obesidade grau I & 05 & 10,0 & 06 & 12,0 & \\
\hline
\end{tabular}


No que se refere à comparação da qualidade de vida (QV) dos grupos estudados, constatou-se, que os indivíduos do grupo com hanseníase apresentaram todos os oitos domínios da qualidade de vida abordados no questionário SF-36 significativamente pior ( $\mathrm{p}<0,005)$ em relação ao grupo de indivíduos sem histórico de hanseníase. Quanto à média de escores dos domínios QV do $\mathrm{GH}$, os escores mais baixos foram: os aspectos físicos (escore = 20,00); dor (escore = 38,66); aspectos emocionais (escore $=41,23$ ); estado geral da saúde (escore $=43,64)$ e capacidade funcional (escore $=49,40)($ Tabela 2$)$.

Tabela 2: Comparação da qualidade de vida dos grupos hanseníase e controle. São Luís - MA, 2017.

\begin{tabular}{llll}
\hline \multicolumn{1}{c}{ Domínios } & \multicolumn{1}{c}{$\begin{array}{c}\text { Grupo Hanseníase } \\
\text { Média } \pm \text { DP (Min.-Max.) }\end{array}$} & $\begin{array}{c}\text { Grupo Controle } \\
\text { Média } \pm \text { DP (Min.-Max.) }\end{array}$ & p-valor** \\
\hline Capacidade funcional & $49,40 \pm 32,914(0-100)$ & $89,50 \pm 13,296(40-100)$ & 0,00000 \\
Aspectos físicos & $20,00 \pm 33,503(0-100)$ & $79,50 \pm 27,521(0-100)$ & 0,00000 \\
Dor & $38,66 \pm 22,834(0-100)$ & $69,88 \pm 17,969(41-100)$ & 0,00000 \\
Estado geral da saúde & $43,64 \pm 22,359(5-97)$ & $68,12 \pm 16,365(30-100)$ & 0,00000 \\
Vitalidade & $52,80 \pm 22,250(10-90)$ & $71,70 \pm 19,420(20-100)$ & 0,00002 \\
\hline Aspectos sociais & $62,62 \pm 31,859(12-100)$ & $89,62 \pm 15,192(37-100)$ & 0,00000 \\
\hline Aspectos emocionais & $41,23 \pm 43,392(0-100)$ & $86,52 \pm 23,516(33-100)$ & 0,00000 \\
\hline Saúde mental & $64,38 \pm 24,008(8-100)$ & $81,48 \pm 14,751(40-100)$ & 0,00004 \\
\hline
\end{tabular}

\section{Discussão}

Observou-se que os oitos domínios da qualidade de vida dos indivíduos com hanseníase apresentaram-se significativamente com piores escores quando comparado aos indivíduos sem hanseníase.

Sobre este aspecto, Rodini et al. (2010) indicam que quando não tratada precocemente, a hanseníase pode alterar diretamente a qualidade de vida dos indivíduos acometidos, gerando grande impacto físico, social e psicológico. Assim, a qualidade de vida dos indivíduos com hanseníase pode ser comprometida devido os estigmas e sequelas da doença como: deficiência física, deformidades, distúrbios psicológicos, dependência econômica e exclusão social. Entre as formas clínicas da doença, os indivíduos mutibacilares são os mais afetados, apresentando acometimentos de leve à muito grave, enquanto os paubacilares podem apresentar-se sem nenhum acometimento ou com acometimento moderado. No entanto, independentemente do tipo clinico da 
doença, sugere-se que os pacientes hansênicos tendem a apresentar algum grau de prejuízo à qualidade de vida (MARTINS; TORRES; OLIVEIRA, 2008; MOSCHIONI et al., 2010).

Salienta-se que em cinco $(62,50 \%)$ dos oitos domínios do SF-36, o GH apresentou média de escore menor que 50, sendo os mais afetados os relacionados aos aspectos físicos, dor, aspecto emocional, estado geral da saúde e capacidade funcional.

Ciconelli et al. (1999) indica que os componentes da qualidade de vida referente à capacidade funcional, aspectos físicos, dor e estado geral de saúde encontram-se diretamente relacionados aos fatores físicos impostos pela doença avaliada, neste caso, a hanseníase. Já os demais componentes abordados pelo questionário SF-36, estaria mais intimamente associado aos fatores mentais que poderiam ser prejudicados pela doença. Neste sentido, destaca-se que dos cinco domínios mais afetados nos pacientes do presente estudo, quatro estão diretamente vinculados aos fatores físicos da hanseníase.

$\mathrm{O}$ aspecto físico, domínio em que os pacientes da presente pesquisa obtiveram menor média de escore, avalia as limitações na forma e quantidade de trabalho (CICONELLI, 1999). De forma similar, o estudo de Araújo et al. (2016) que avaliou a QV de 59 pacientes com hanseníase, também encontrou os menores escores no domínio de aspecto físico. Provavelmente este fato é justificado por tratar-se de um domínio significativamente relacionado às incapacidades físicas (MARTINS, 2009).

Sobre este aspecto, evidencia-se que aproximadamente dois a três milhões de indivíduos hansênicos tenham algum grau de comprometimento físico decorrente da doença. Aproximadamente $20 \%$ dos pacientes com hanseníase ou tratados para a doença podem apresentar incapacidades físicas e restrições psicossociais. No Brasil, sugere-se que $23 \%$ dos pacientes hansênicos apresentam algum tipo de incapacidade após a alta (GONÇALVES; SAMPAIO; ANTUNES, 2009; RAMOS; SOUTO, 2010).

Gaudenci et al. (2015) afirmam que o alto grau de incapacidade física do paciente hansênicos é o principal responsável pelas maiores consequências da doença, pois atinge diretamente a produtividade laboral, as atividades da vida diária e as relações interpessoais, como também desencadeia transtornos psicossociais e queda na QV de seus portadores, com prejuízo de natureza social, econômica e psicológica.

Neste mesmo sentido, o domínio referente à capacidade funcional da qualidade de vida refere-se às limitações físicas e o quanto essas limitações interferem na capacidade física (CICONELLI, 1999). Em pacientes com hanseníase, a capacidade física pode ser prejudicada 
devido as próprias consequências comum à doença, como espessamento do nervo, e sensibilidade alterada, que em geral causam incapacidades funcionais envolvendo pincipalmente os olhos, mãos e pés (RODRIGUES et al., 2015).

Salienta-se que as alterações físicas resultantes da hanseníase alteram a aparência e causam limitações severas no desempenho das atividades de vida diária dos indivíduos com diagnóstico desta doença, já que se trata de sequelas muitas vezes desfigurantes, mutilantes e incapacitantes (DIAS; CYRINO; LASTÓRIA, 2007).

Segundo Nascimento et al. (2011), muitos pacientes que descobrem a doença, já apresentam deformidade e incapacidades motoras e ou sensitivas, que poderiam ser evitadas se o diagnóstico e o tratamento fossem precoces. Brakel (2012) acrescenta que esses indivíduos, mesmo após o tratamento da hanseníase, apresentam um risco substancial de ter deficiência e deterioração física.

O domínio dor também se mostrou significativamente afetado pela hanseníase. Segundo Ciconelli (1999), esse domínio é referente à intensidade e como a dor é capaz de interferir no desenvolvimento das atividades de vida diária - AVD’s. Nos pacientes com hanseníase, a dor é uma das sintomatologias mais frequentes, principalmente devido ao acometimento dos nervos (PUCCI et al., 2011). Sobre este dado, Victor et al. (2016) realizou um estudo para avaliar a QV através do questionário WHOQOL-BREF em 260 pacientes com hanseníase, constatando que 75,0\% deles sentiam dor, sendo que estes apresentaram os escores de qualidade de vida menores nos domínios saúde física com $(p<0,0001)$, saúde psicológica $(p=0,001)$ e ambiental $(p=0,001)$.

A influência da hanseníase sobre o domínio referente aos aspectos emocionais da qualidade de vida pode estar relacionada aos sintomas e sequelas da doença, caracterizada por forte dores e incapacidades que podem interferir no desenvolvimento normal do trabalho e das atividades de vida diária. Dado confirmado pelo estudo de Araújo et al. (2016), em que o aspecto emocional de indivíduos com hanseníase também obteve média de escore inferior a 50.

O domínio denominado estado geral de saúde, que avalia a percepção dos indivíduos quanto a sua própria saúde, também foi significativamente menor em pacientes com hanseníase. Sugere-se que isto pode estar associado ao fato de que a hanseníase é uma doença infectocontagiosa crônica, e incapacitante, que mesmo possuindo cura, ainda envolve grande estigma e preconceito, o que acaba influenciando a percepção dos doentes quanto a sua própria saúde (CICONELLI, 1999; MOSCHIONI et al., 2010). Assim, é comum nestes pacientes sentimentos como medo, vergonha, culpa, exclusão social, rejeição e raiva (BAIALARDI, 2007). 
Dentre os oito domínios da QV, os indivíduos com hanseníase estudados obtiveram maiores escores nos relacionados à vitalidade, aspectos sociais e saúde mental. No entanto, ressaltase que foram identificadas diferenças significativas entre o GH e o GC em relação à estes domínios também.

A vitalidade analisa o nível de energia e fadiga de cada indivíduo (CICONELLI, 1999). Considera-se que devido às incapacidades que a hanseníase pode causar, o indivíduo desenvolve déficits funcionais que o torna mais propenso à fadiga. Sobre este aspecto, diferente deste estudo, Lopes Neto et al. (2015), realizou uma pesquisa com 57 indivíduos com hanseníase e indicou a vitalidade como o domínio da qualidade de vida com o pior escore. Destaca-se que a amostra desta pesquisa é constituída por adultos jovens, o que pode ter levado à uma maior escore neste domínio.

O domínio aspectos sociais do questionário SF-36 avalia a participação dos indivíduos em grupos sociais e se esta encontra-se comprometida por algum problema de saúde (CICONELLI, 1999). Considera-se este domínio essencial na avaliação de paciente hansênicos, já que se trata de uma doença que ao longo da história foi discriminada (CICONELLI, 1999; MOSCHIONI et al., 2010). Mesmo nos dias atuais, o paciente com hanseníase ainda se encontra cercado de forte estigma social que resulta em discriminação social, isolamento, perda da capacidade laborativa alteração da sua autoestima e prejuízos econômicos (AMARAL; LANA, 2008; SÁ; PAZ, 2007).

O domínio saúde mental avalia a presença de angústia e bem-estar psicológico (CICONELLI, 1999). Este domínio também pode estar relacionado aos vários fatores já supracitados da hanseníase, tanto pelo preconceito ainda atrelado a essa doença, quanto por se tratar de uma doença que acarreta aparecimento de manchas visíveis pigmentadas ou discrômicas; formação de placas que ocorre quando as lesões se estendem em superfície por vários centímetros; infiltrações que ocorre devido ao aumento da espessura e consistência da pele, acompanhado às vezes de eritema discreto, além de pápulas ou nódulos que evoluem deixando cicatrizes (BARBIERI; MARQUES, 2009). Assim, o diagnóstico de hanseníase pode levar a reações psicológicas confusas, que refletem em afastamento social, vergonha de si, medo da morte etc. (SILVEIRA et al., 2014).

Faz-se necessário salientar que muitas vezes o indivíduo hansênicos sofre o preconceito e discriminação devido à falta de informação, já que se sabe que ao realizar o tratamento adequadamente, o indivíduo não é mais capaz de transmitir a doença. Assim, os pacientes com hanseníase em tratamento assistido podem conviver normalmente com sua família, seus colegas de trabalho e amigos (RODINI et al., 2010). 
Evidencia-se ainda que o diagnóstico de hanseníase deve ser informado ao paciente de forma similar aos diagnósticos de outras doenças crônicas curáveis. Cabe a equipe de saúde efetivar uma abordagem apropriada tanto no momento do diagnóstico, quanto no decorrer do tratamento da doença e após a alta por cura. Tal abordagem deve considerar o impacto psicológico da doença para o paciente e para seus familiares, visando maior adesão aos tratamentos e diminuição do estigma social (BRASIL, 2010).

Desta forma, evidencia-se o importante impacto da hanseníase na qualidade de vida dos participantes deste estudo. Isso indica a necessidade de realizar assistência à saúde para esses indivíduos de maneira holística, através de uma equipe interdisciplinar, aonde se pode incluir o fisioterapeuta, profissional habilitado a atuar em todos os níveis de atenção à saúde, abrangendo a prevenção, proteção, promoção e reabilitação de indivíduos (BARAÚMA et al., 2008).

\section{Conclusão}

Apesar da homogeneidade entre os grupos estudados quanto à idade, gênero e IMC, constatou-se que os indivíduos com hanseníase apresentaram os oito domínios referente à qualidade de vida com escores significativamente menores que os indivíduos do grupo controle, indicando que a hanseníase causou impacto em todos estes domínios,

Desta forma, esses resultados são importantes para que os profissionais de saúde envolvidos no tratamento desses indivíduos tenham consciência que a hanseníase é uma doença que envolve fatores físicos, psicológicos, mentais e sociais, devendo-se tratar não apenas os sintomas da doença, mas os indivíduos como todo, ou seja, de forma biopsicossocial, através de uma equipe interdisciplinar.

Destaca-se ainda que os domínios da qualidade de vida afetados pela hanseníase poderiam ser amenizados com o diagnóstico e o tratamento precoce, pois muitos pacientes descobrem a doença tardiamente, já apresentando deformidade e incapacidades motoras e ou sensitivas, que poderiam ser evitadas. Considera-se que pacientes com diagnóstico precoce e tratamento adequado, teriam ausência ou diminuição dessas deformidades e incapacidades e, consequentemente, uma melhor qualidade de vida. 


\section{Referências}

AMARAL, E. P; LANA, F. C. F. Análise espacial da hanseníase na microrregião de Almenara, MG, Brasil. Revista Brasileira de Enfermagem, Brasília, v. 61, p. 701-707, Out.2008.

ARAÚJO, D.A.L. et al., Characteristics of people of quality of life with leprosy in outpatient treatment. Rev Fund Care Online. v.8: p.5010-5016, out-dez. 2016. Disponível em $<$ http://www.seer.unirio.br/index.php/cuidadofundamental/article/view/4732> Acesso em :06 de jun 2016.

AVELLEIRA, J. C. R. et al. History of leprosy in Rio de Janeiro. Anais brasileiros de dermatologia, v. 89, n. 3, p. 515-518, 2014.

BAIALARDI, K. S. O estigma da hanseníase: relato de experiência em grupo com pessoas portadoras. Hansenologia Internationalis. v. 1, n. 32, p. 27-36, Mar. 2007.

BARAÚNA, M. A.et al., A importância da inclusão do fisioterapeuta no Programa de Saúde da Família. Fisioter. Bras; v.9 p.64-69, jan.-fev. 2008.

BARBIERI, C. L. A.; MARQUES, H. H. S. Hanseníase em crianças e adolescentes: revisão bibliográfica e situação atual no Brasil. Pediatria, v. 31, n.4, p. 281-90. 2009

BARBOSA, F. P. S. et al., Incapacidades Neurológicos provocadas pela hanseníase em uma unidade de saúde do município de Anápolis-GO, entre 2011 e 2013, Revista Educação Saúde, v.4, n.2, p.3-10, 2016

BRAKEL, W. H. et al. Disability in people affected by leprosy: the role of impairment, activity, social participation, stigma and discrimination. Glob Health Action, v.5 mar/jun.2012.Disponível em:</https://www.ncbi.nlm.nih.gov/pmc/articles/PMC340206 9/> Acesso em: 18 set. 2016.

BRASIL, Ministério da Saúde. Secretária de Vigilância em Saúde. Anotações. Brasília: Ministério da Saúde, 2014.

BRASIL. Ministério da Saúde. Doenças infecciosas e parasitárias: guia de bolso. 8 ed. Brasília: Ministério da Saúde, 2010.

BRASIL. Ministério da Saúde. Eliminar a hanseníase é possível: um guia para municípios. Brasília: Ministério da Saúde, 2015a.

BRASIL. Ministério da Saúde. Secretária de Vigilância em Saúde. Departamento de Vigilância das Doenças transmissíveis. Brasília: Ministério da Saúde, 2015b.

BRITO, A. L. et. al. Tendência temporal da hanseníase em uma capital do Nordeste do Brasil: epidemiologia e análise por pontos de inflexão, 2001 a 2012. Rev. bras. epidemiol, São Paulo, v.19, n.1, p.194-204, Jan/mar.2016. 
CICONELLI, R. M. et al. Tradução para a língua portuguesa e validação do questionário genérico de avaliação de qualidade de vida SF-36. Rev Bras Reumatol, v. 39, n.3, p. 143-150, 1999.

CICONELLI, R. M. Medidas de avaliação de qualidade de vida. Rev. Bras. Reumatologia, v. 43, n. 2, p.9-13, 2003.

DIAS, A; CYRINO, E. G; LASTÓRIA, J. C. Conhecimentos e necessidades de aprendizagem de estudantes de fisioterapia sobre a hanseníase. Hansenologia internationalis, v.32, n.1, p.9-18,2007. GAUDENCI, E. M. et al. Qualidade de Vida, Sintomas Depressivos e Incapacidade Física de Pacientes com Hanseníase. Hansen Int. v. 40, n. 2, p. 48-58, 2015.

GONCALVES, S. D.; SAMPAIO, R. F.; ANTUNES, C. M. F. Fatores preditivos de incapacidades em pacientes com hanseníase. Rev. Saúde Pública, v.43, n.2, p. 267-274, 2009.

HENRY, B. et al. Development of the Cystic Fibrosis Questionnaire (CFQ) for assessing quality of life in pediatric and adult patients. Qual Life Res, v. 12 n. 1, p. 63-76, 2003.

LASTÓRIA, J. C.; ABREU, M. A. M. M. Hanseníase: diagnóstico e tratamento. Diag.

Tratamento, v.17, n. 4, p. 173-9, 2012. Disponível em <http://files.bvs.br/upload/S/14139979/2012/v17n4/a3329.pdf>. Acesso em: 20 set. 2016.

LOPES NETO, D. et al. Avaliação da qualidade de vida em pacientes com hanseníase: uso do Medical Outcomes Study 36. Rev. Bras. Pesq. Saúde, Vitória, v. 17, n.1, p. 6-10, jan-mar, 2015 MARTINS, B. D. L.; TORRES, F. N.; OLIVEIRA, M. L. W. Investigação Clínica, Epidemiológica, Laboratorial e Terapêutica. Impacto na qualidade de vida em pacientes com hanseníase: correlação do Dermatology Life Quality Index com diversas variáveis relacionadas à doença. Anais. Rio de Janeiro: Brasileiros de Dermatologia, p.39-43. 2008.

MARTINS, M. A. Qualidade de Vida em Portadores de Hanseníase. 2009 .97 F. Dissertação (Mestrado em psicologia) - Universidade Católica Dom Bosco, Campo Grande. 2009.

MONTEIRO, L. D. et al. Incapacidades físicas em pessoas acometidas pela hanseníase no período pós alta da poliquimioterapia em município no norte do Brasil, Cad. Saúde Pública, Rio de Janeiro, v.29, n.5, p.909-20, Maio,2013.

MOSCHIONI, C. et al., Risk factors for physical disability at diagnosis of 19,283 new cases of leprosy. Revista da Sociedade Brasileira de Medicina Tropical, v. 43, n.1, p.19-22, 2010. NASCIMENTO, G.R.C. et al. Ações do enfermeiro no controle da hanseníase; Revista eletrônica enfermagem. v. 13, n.4, p.743-50, out/dez.2011.Disponível em <http://www.fenufg.br/revista/v13/n4/v13n4a20.htm> acesso em :18 de set2016 
OPROMOLLA, P. K. et al. Controle da hanseníase no Estado de São Paulo: análise histórica. Revista de Saúde Pública, v. 45, n. 1, p. 195-203, 2011.

PUCCI, F.H. et al., A dor no paciente com hanseníase, Rev Dor. São Paulo, jan-mar; 2011.

RAMOS, J. M. H.; SOUTO, F. J. D. Incapacidade póstratamento em pacientes hansenianos em Várzea Grande, Estado de Mato Grosso. Rev. Soc.Bras. Med. Trop., Uberaba, v.43, n.3, p.293297. May/June 2010.

RODINI, F. C. B. et al. Prevenção de incapacidade na hanseníase com apoio em um manual de autocuidado para pacientes. Fisioterapia e Pesquisa, São Paulo, v.17, n.2, p.157-66, abr/jun. 2010. RODRIGUES, F. F. et al. Conhecimento e prática dos enfermeiros sobre hanseníase: ações de controle e eliminação. Rev. Bras. Enferm, v.68, n.2, p.297-304, 2015.

SÁ, A. M. M; PAZ, E. P. A. O cotidiano de ser hanseniano: um estudo de enfermagem. Hansenologia Internationalis. v. 32, n. 1, p. 49-55, Jan. 2007.

SARAIVA, A. P.; V.;NÓBREGA, M. S. G. Avaliação da qualidade de vida em pacientes com síndrome de Down na APAE - João Pessoa - Paraíba. PesqBrasOdontopedClinIntegr, João Pessoa, v. 3, n. 2, p. 59-64, jul./dez. 2003.

SILVA, P. H. A. Levantamento epidemiológico dos pacientes assistidos no serviço de referência em hanseníase do município de Campina Grande-PB no período de 2003 a 2012. 2013. 26f. Trabalho de Conclusão de Curso (Graduação em Farmácia) - Universidade Estadual da Paraíba, Campina Grande, 2013.

SILVEIRA, M. G. B. et al. Portador de hanseníase: impacto psicológico do diagnóstico. Psicol Soc., v. 26, n. 2, p.517-527, 2014.

UCHÔA, R. E. M. N. et al. Perfil Clínico e incapacidades físicas em pacientes com hanseníase, Rev. Enf. UFPE online., Recife, 11(Suplemento.3), p.1464-72, Mar., 2017.

VÉRAS, L.S.T. et.al., Avaliação da dor em portadores de hanseníase submetidos à mobilização neural. Fisioterapia e Pesquisa, São Paulo, v.18, n.1, p. 31-6, jan/mar. 2011.

VITORINO, D. F. M. et al. Utilização do SF-36 em ensaios clínicos envolvendo pacientes fibromiálgicos: determinação de critérios mínimos de melhora clínica. Revista Neurociências, v.12, n. 3, p. 147-151, 2004. 